\section{Interactive data entry to ACOVS: A program for the analysis of covariance structures}

\author{
MICHAEL L. FRIENDLY \\ York University, Toronto, Ontario M3J 1P3, Canada \\ WILLIAM R. KRANE \\ Simon Fraser University \\ Burnaby, British Columbia V5A IS6, Canada
}

and

\section{SAMPO V. PAUNONEN \\ University of Western Ontario \\ London, Ontario N6A 5C2, Canada}

ACOVS (Jöreskog, Gravaeus, \& van Thillo, Note 1) is a general computer program for the analysis of covariance structures that is based on a model developed by Jöreskog (1970). It has considerable flexibility and is capable of handling a wide variety of multivariate problems. Typically, the input to ACOVS consists of a card file that has been punched according to the specifications outlined by Jöreskog et al. However, the creation of this input file can prove to be quite tedious, especially when the number of parameters to be estimated is large or when there are several restrictions placed on the parameters. The following is a brief description of an interactive program that has been designed to facilitate data entry to ACOVS. Details on the features of the interactive system are provided by Paunonen, Krane, and Friendly (Note 2).

Description. The interactive program allows a user to create (or modify) a disk file of card images, which is suitable for input to the ACOVS program, by responding to teletype or CRT prompts. These prompts have been written under the assumption that the user has a familiarity with both the model underlying ACOVS and the input requirements of the program. Data input is

This research was supported by Grant $A 7117$ awarded to the second author and Grant $\mathbf{A 8 6 1 5}$ awarded to the first author from the Natural Sciences and Engineering Research Council of Canada. expedited by a routine that can be used to rapidly generate a matrix of pattern values or starting-point values when sequences of values are repeated within the matrix. An editing routine allows file modifications to be made to new or existing files, line by line or character by character.

Input. Input to the program consists of user replies to selective teletype prompts that follow from previous responses. Some examples of such prompts are "ORDER OF INPUT MATRIX = " and "ENTER ROW VECTOR OF STANDARD DEVIATIONS." After completing the initial data entry or upon accessing a previously created data set, the user may examine and modify the file of card images.

Output. The program creates a disk file of 80-column card images formatted according to the requirements of the ACOVS program detailed by Jöreskog et al. (Note 1). Currently, it can accommodate data sets up to 500 records in length.

Computer and Language. The interactive program is written in FORTRAN IV for use on a DEC 10 system.

Availability. A source listing and sample run can be obtained free of charge by writing to Sampo Paunonen, Department of Psychology, University of Western Ontario, London, Ontario N6A 5C2, Canada. Requests for a copy of the program should be accompanied by an unlabeled nine-track tape. The ACOVS program is not supplied.

\section{REFERENCE NOTES}

1. Jöreskog, K. G., Gruvaeus, G. T., \& van Thillo, M. ACOVS: A general computer program for the analysis of covariance structures (Research Bulletin 70-15). Princeton, N.J: Educational Testing Service, 1970.

2. Paunonen, S. V., Krane, W. R., \& Friendly, M. L. A user's guide to an interactive program for the analysis of covariance structures (Research Bulletin 459). London, Ontario: University of Western Ontario, 1978.

\section{REFERENCE}

Jöreskog, K. G. A general method for analysis of covariance structures. Biometrika, 1970, 52, 239-251.

(Accepted for publication March 18, 1980.) 